\title{
La preposición ' $a$ ' en la expresión escrita de los alumnos egipcios de ELE
}

\author{
Rasha Ali ABDEL EL AZIM \\ Departamento de Lengua Española / Facultad de Lenguas y Traducción \\ Universidad de Misr para la Ciencia y Tecnología. Egipto \\ rasha.ali24@gmail.com
}

Recibido: diciembre 2013

Aceptado: julio 2014

\begin{abstract}
RESUMEN
El presente trabajo tiene dos objetivos principales: manifestar la importancia del análisis contrastivo con el fin de entender las dificultades que llegan a tener los alumnos al poner en práctica correctamente algunas estructuras en segunda lengua (L2) y eso debido en gran parte a la diferencia del sistema de su lengua materna, pero también a la sutilidad de la oposición de unas reglas en L2, y por otro lado reconocer a través del análisis de errores las posibles razones que llevan al uso erróneo de algunas estructuras. En nuestro estudio concretamos un caso determinado: el sistema preposicional, fijándonos en el uso de la preposición $a$ en la expresión escrita de alumnos egipcios de ELE. Para ello empleamos dos metodologías: el análisis contrastivo y el análisis del error. El análisis contrastivo da a conocer el sistema preposicional en la lengua materna del grupo investigado, que será el árabe, y la lengua objeto que se está aprendiendo, el español. Nos centramos en el valor único que posee la preposición árabe $i l a$ 'a' y la preposición española $a$ respectivamente.

Con esta base iniciamos una investigación empírica sobre la preposición $a$ en la expresión escrita de alumnos egipcios de ELE de nivel intermedio y superior. Las herramientas principales han sido un cuestionario sobre el historial de las lenguas extranjeras de los alumnos y una tarea de redacción.
\end{abstract}

Palabras clave: Aprendizaje de la lengua, errores, expresión escrita, interferencias, lingüística contrastiva.

\section{The preposition 'a' in written expression of Egyptian students of Spanish language}

\begin{abstract}
This present investigation has two main objective: to demonstrate the importance of contrastive analysis that allows us to understand the difficulties that students suffer when they try to use correctly some structures in L2 due to the difference in their native language system or due to the subtlety of opposition of some rules in L2 which in our case would be the prepositional system, studying the case of the preposition $a$ 'to' in written expression of Egyptian students of Spanish language, and to shed light upon possible reasons that lead students to make fossilized errors when using the preposition ' $a$ ' in Spanish.
\end{abstract}


The theoretical framework of the thesis is based mainly on two methodologies: contrastive analysis and error analysis. The contrastive analysis reveals the system of preposition in both the subjects' native language, which is Arabic, and the subjects' language of study, which is Spanish. We focus on the unique value of the preposition ila 'to' and the preposition $a$ 'to' respectively.

With this in mind, we begin an empirical study of the preposition $a$ 'to' using written samples taken from intermediate and advanced leveled Egyptian students of Spanish as a foreign language. Data were collected from a survey on the language background of the students and a writing task.

Keywords: Language learning-Errors- written production- Interferences- Contrastive linguistics.

\section{La préposition «a » dans l'expression écrite des élèves égyptiens d'ELE}

\section{RÉSUMÉ}

Le présent travail vise deux objectifs principaux. Le premier est de montrer l'importance de l'analyse contrastive dans la compréhension des difficultés probables des étudiants à l'heure d'appliquer de façon correcte certaines structures de la langue seconde (L2), en raison en grande partie de la différence du système de la L2 par rapport à celui de sa langue maternelle, mais aussi aux subtilités de l'opposition de certaines règles en L2. Le second est d'identifier, à travers l'analyse d'erreurs, les causes possibles de l'usage erroné de certaines structures. Cette étude se concentre autour d'un cas concret défini: le système prépositionnel. Elle se centre particulièrement sur l'utilisation de la préposition « $\mathrm{a}$ » dans l'expression écrite d'étudiants égyptiens d'espagnol langue étrangère (ELE). Pour cela, deux méthodologies sont appliquées : l'analyse contrastive et l'analyse d'erreurs. L'analyse contrastive permet d'identifier le système prépositionnel de la langue maternelle du groupe étudié, c'est-à-dire l'arabe, et de celui de la langue seconde, l'espagnol. Le travail se focalise sur la valeur unique que possèdent respectivement la préposition arabe « ila » et l'espagnole « a ».

Sur cette base se construit une recherche empirique sur l'usage de la préposition «a» par des étudiants égyptiens de ELE - niveaux intermédiaire et supérieur - dans la compétence d'expression écrite. Les outils principaux pour l'investigation ont été un questionnaire sur l'historique des langues des étudiants et un exercice de rédaction.

Mots-clés: Apprentissage de la langue-Erreurs- Expression écrite- Interférenceslinguistique contrastive.

SUMARIO: Introducción 1. Análisis contrastivo: la preposición ila 'a' en árabe y la preposición $a$ en castellano.1.1. El significado de la preposición $a$. 1.2. El significado de la preposición ila 'a'. 1.3. Análisis contrastivo de la novela egipcia Taxi y su traducción en español.1.3.1. Comentario sobre la correspondencia de la preposición ila 'a' en la versión española de Taxi. 1.3.2. Comentario sobre la correspondencia de la preposición $a$ en la versión árabe de la novela Taxi. 2. Análisis de errores. 2.1. Material de la muestra. 2.2. Toma de datos. 2.3. Descripción del corpus. 2.4. Presentación de los resultados del análisis de errores 3. Conclusión. 4. Bibliografía. 


\section{INTRODUCCIÓN}

Investigar con base científica sobre el desarrollo de una segunda lengua, nos obliga a aludir a la distinción que establece Krashen (1982: 10-12) entre adquisición y aprendizaje basada en la diferencia entre lo inconsciente y lo consciente respectivamente. El aprendiente tiene preestablecido el modelo de su lengua materna como una referencia inconsciente. Normalmente no somos conscientes de la realidad que estamos adquiriendo una lengua, pero sí somos conscientes de que utilizamos la lengua para comunicarnos. Cuando se aprende una L2, se introduce un conocimiento nuevo y consciente que sigue a lo que él llama "orden natural". Krashen supone que las estructuras gramaticales de lengua extranjera no pertenecen a un proceso de aprendizaje sino de adquisición con un orden predeterminado, de la misma manera que existe un orden en la adquisición de reglas de la lengua materna. Por otra parte, da a conocer al Modelo del Monitor: todos llevamos dentro un monitor, constituido por las reglas del sistema fonológico, léxico o gramatical, que nos permite corregir y modificar nuestras producciones. Se considera como responsable de la edición de las producciones del sujeto y único vínculo entre el conocimiento adquirido y aprendido.

Este enfoque revolucionario de los 80 manifiesta el papel del aprendiz en el proceso adquisición/ enseñanza de la lengua. Hemos hecho esta síntesis histórica ya que a nuestro juicio nos permite entender mejor la evolución de la concepción del error, objeto de nuestro estudio, centrándonos en dos métodos que trataremos en unas líneas más adelante: Análisis Contrastivo (AC) y Análisis de Errores (AE).

La palabra "aprendizaje" implica la aparición de otro término "el error". Los errores se relacionan con nuestras actividades vitales en general y de hecho la mayoría pensamos que sin cometer errores no estamos aprendiendo.

El interés por los errores de la lengua, dentro del proceso de adquisición de la misma, se fue incrementando en el siglo XX, abriendo camino hacia la aparición de unas teorías pioneras con aportaciones muy significativas. Antes de exponerlas, resulta conveniente definir primero el significado del error.

Según James, un error de lengua es "unsuccessful bit of language", es decir, poco éxito del lenguaje (1981: 1). Fernández López (1995: 204) define el error como "cualquier transgresión involuntaria de la norma". Y la norma ¿qué es?: "un sistema de reglas que definen lo que se debe elegir entre los usos de una lengua determinada, si se quiere estar de acuerdo con cierto ideal estático y sociocultural, o lo que es normal común en una sociedad dada." El Marco Común Europeo de Referencia para las Lenguas (1991: 153) define el error como una representación deformada de la competencia meta que en este caso desarrolla características distintas de las normas de la segunda lengua.

La aplicación de las teorías lingüísticas y psicológicas al aprendizaje de la lengua, según comentan Corder y otros lingüistas más (1981: 5), han aportado nuevas dimensiones al tratar el error: se ha difundido la creencia de que los errores son el resultado de la interferencia de hábitos de primera lengua (L1) en L2. El AC 
defendía la idea de que los alumnos recurren a su conocimiento de L1 para facilitar el aprendizaje de L2, considerado en este caso como una transferencia positiva. El problema surge cuando un elemento de L2 no tiene equivalencia en L1, lo que dará lugar a la transferencia negativa, es decir, al error. Weinreich (1968:1) afirma que cuanto mayor es la diferencia en el sistema lingüístico entre dos lenguas o dos dialectos de una lengua, mayor serán las dificultades del aprendizaje, y como consecuencia, la interferencia ocupa un espacio más amplio.

El AE nació debido a las limitaciones del $\mathrm{AC}$, ya que el hecho de contrastar dos sistemas lingüísticos no debería ser el único factor que afecta al proceso del aprendizaje de lenguas. Corder (1967:10) provocó un cambio significativo en la orientación del estudio del error, deteniéndose ante las producciones reales de los hablantes de L2, realzando la importancia de las estrategias de aprendizaje en vez de contrastar L1 y L2. A partir de aquel momento comienza una nueva etapa de investigaciones tanto en la teoría como en la práctica que reformula la conceptualización del error.

Las fases del trabajo dentro del AE se basan principalmente en la propuesta de Corder (1967:65) consistente en identificar los errores, describir los errores y explicar aquellos rasgos que desvían al alumno de la lengua meta. En la misma línea, Santos Gargallo (2004:397-398) comenta que el objetivo del análisis debe tener en cuenta los siguientes criterios: la lengua materna, la extensión de la muestra, la habilidad lingüística, la extensión del análisis y la periodicidad en la recogida de datos. Criterios que serán aplicados en la parte empírica de nuestra investigación.

A continuación expondremos nuestro trabajo que se centra en los errores de la preposición $a$ en la expresión escrita de alumnos egipcios de ELE aplicando dos metodologías que se complementan entre sí: $\mathrm{AC}$ y AE. Comenzamos con la parte contrastiva.

\section{ANÁliSIS CONTRASTIVO: LA PREPOSICIÓN ILA 'A' EN ÁRABE Y LA PREPOSICIÓN 'A' EN CASTELLANO}

Este apartado se compone de dos partes: la primera presenta la teoría sobre el valor de la preposición $a$ en español y la de ila 'a' en árabe, mientras que la segunda contrasta la aparición de la preposición $a$ en una novela egipcia llamada taxi y la preposición ila en la traducción española de la misma para subrayar los diferentes tipos de correspondencias y ofrecer un inventario que nos ayude a entender mejor los motivos de los errores de la preposición $a$ en la expresión escrita de alumnos árabes.

\subsection{El significado de la preposición 'a'}

Según la gramática de la RAE (2010: 565), el significado de las preposiciones es relacional, por lo cual se presenta como el significado que corresponde a su término. La preposición a introduce complementos de destino (voy a Madrid) y de 
término (salimos a la terraza). A dichos rasgos de ubicación se le añade el de dirección u orientación, como, por ejemplo, la casa da al sur.

Para determinar el valor de las preposiciones, Llorch (1995:222) ha utilizado el rasgo dinámico (en el que interviene el movimiento, ya sea físico o figurado). Las preposiciones que pertenecen al grupo dinámico son $a$, contra, hacia, hasta y para e implican acercamiento a la noción designada por su término, mientras que las que aluden a alejamiento serían de y desde, y la intermedia por. Las preposiciones $a$, hasta y contra, que denotan acercamiento, indican atingencia al límite, mientras que hacia y para señalan mera dirección hacia él.

En los casos de $a$ y hasta, el primero alude solamente al destino mientras que el segundo expresa la distancia que conduce a él:

1. Voy a la universidad

2. Voy hasta la universidad.

Trujillo (1971: 265) clasifica las preposiciones en dos grupos, dependiendo de la presencia o de la ausencia del sema movimiento:

a) Caracterizadas por el sema movimiento: a, contra, de, desde, hacia, hasta, para, por.

b) Caracterizadas por el sema no movimiento: ante, bajo, con, en, entre, según, sin, sobre, tras.

\subsection{El significado de la preposición ila 'a'}

Según Hussein Abid (2008:329), la preposición ila 'a' indica el final del destino, sea este punto final una persona o una cosa o un lugar:

3. Zahab ila al madrasa.

Ir[PAS 3a PS] a el colegio.

'Ha ido al colegio.'

Después de esta aclaración del significado de la preposición en las dos lenguas, resulta conveniente y útil subrayar más ejemplos en los que mientras que la lengua árabe optaría por la preposición ila 'a', la lengua española optaría por la equivalencia $a$ o por otra distinta y viceversa. Este sería el eje temático de la siguiente sección.

\subsection{Análisis contrastivo de la novela egipcia Taxi y su traducción en español}

En el presente apartado hacemos un análisis contrastivo, utilizando la versión original de la novela egipcia titulada, Taxi, de El Khamissi (2006), y su traducción al español, de A. Cano y K. Musa (2009). El objetivo de este análisis es subrayar el sistema preposicional tanto en árabe como en español, manifestando las semejanzas y las diferencias del uso de la preposición ila 'a' y su equivalencia en español y la correspondencia de la $a$ en árabe. Esto nos ayuda a darnos cuenta de los posibles errores que puedan cometer los alumnos egipcios de ELE al hacer uso de dicha preposición. Se divide esta sección en tres partes: en la primera exponemos tablas estadísticas de la correspondencia de la preposición en ambos 
idiomas y en las otras dos comentamos y explicamos algunos casos relevantes del árabe al español y viceversa.

La obra original Taxi ofrece una imagen realista de la sociedad egipcia actual. Es una recopilación de charlas que ha mantenido el protagonista con unos taxistas de El Cairo, tratando diferentes temas cotidianos tanto políticos como sociales de algunas cuestiones alarmantes para la sociedad. El estrato social de los taxistas incluye a personas analfabetas y otras con educación básica, universitaria o incluso con estudios de posgrado, que ejercen esta profesión con el fin de mejorar sus ingresos económicos. Esta variedad social ha marcado el estilo de la narración que mezcla el registro culto y el coloquial: se utiliza el lenguaje coloquial en las conversaciones con los taxistas mientras que el registro culto se emplea en las descripciones previas o intervenciones realizadas por el autor. Esta característica del estilo narrativo nos ha obligado a tenerla en cuenta a la hora de realizar nuestro análisis como muestran las tablas de abajo.

El trabajo contrastivo ha seguido unas líneas principales: primero identificar los casos de la preposición $a$ en la versión española de la novela Taxi y los de ila 'a' en la versión original árabe; segundo, identificar la correspondencia entre el castellano y el árabe, y tercero observar la distribución en los respectivos géneros. Se dan los resultados en las tablas del 1-4:

\begin{tabular}{|l|l|l|l|l|l|}
\hline Totales & A & En & Hasta & Hacia & [otra ] \\
\hline Ila & 50 & 23 & 10 & 10 & - \\
Ntot $/ 100 \%$ & $53.76 \%$ & $24.73 \%$ & $10.75 \%$ & $10.75 \%$ & \\
\hline
\end{tabular}

Tabla 1. Correspondencia de la preposición ila 'a'

Cotejando la versión árabe de la novela, Taxi, con la traducción al español encontramos que la preposición ila 'a' coincide con la preposición $a$ en un $53 \%$ de los casos, mientras que equivale a la preposición en con un porcentaje del $24 \%$ frente a las dos últimas hasta y hacia con un porcentaje del 10\%:

\begin{tabular}{|c|c|c|c|c|c|c|c|c|c|}
\hline Totales & $\varnothing$ & Ila & $\begin{array}{l}\text { Fi } \\
\text { 'en' }\end{array}$ & $\begin{array}{l}\text { Min } \\
\text { 'de' }\end{array}$ & $\begin{array}{l}\mathrm{Li} \\
\text { 'a' } \\
\text { 'para }\end{array}$ & $\begin{array}{l}\text { Alaa } \\
\text { 'sobre' }\end{array}$ & $\begin{array}{l}\text { Bi } \\
\text { 'por' } \\
\text { / 'con' }\end{array}$ & $\begin{array}{l}\text { An } \\
\text { 'alejánd } \\
\text { ose' }\end{array}$ & otra \\
\hline $\begin{array}{l}\text { A } \\
\text { Ntot/100 } \\
\%\end{array}$ & $\begin{array}{l}351 \\
55.62 \\
\%\end{array}$ & $\begin{array}{l}50 \\
8.7 \\
\%\end{array}$ & $\begin{array}{l}72 \\
11.4 \\
1 \%\end{array}$ & $\begin{array}{l}15 \\
2.37 \\
\%\end{array}$ & $\begin{array}{l}77 \\
12.2 \\
0 \%\end{array}$ & $\begin{array}{l}38 \\
6 \\
\%\end{array}$ & $\begin{array}{l}20 \\
3.16 \\
\%\end{array}$ & $\begin{array}{l}4 \\
0.6 \\
\%\end{array}$ & $\begin{array}{l}4 \\
0.6 \\
\%\end{array}$ \\
\hline
\end{tabular}

Tabla 2. Correspondencia de la preposición $a$ en los dos registros: cultopopular 
El porcentaje total de la correspondencia de la preposición $a$ en la versión árabe de la novela, objeto de comparación, muestra ausencia de algún tipo de correspondencia con un porcentaje que llega al 55\% mientras que la correspondencia con las preposiciones $f i$ 'en', y $L i$ 'a/ para' es del $11 \%$ y el $12 \%$, respectivamente. La equivalencia con la preposición ila 'a' ocupa un porcentaje menor que los demás $8 \%$. En conclusión el porcentaje de errores por omisión registra el nivel más alto.

\begin{tabular}{|l|l|l|l|l|l|l|l|l|l|}
\hline $\begin{array}{l}\text { Registro } \\
\text { culto }\end{array}$ & $\varnothing$ & Ila & $\begin{array}{l}\text { Fi } \\
\text { 'en' }\end{array}$ & $\begin{array}{l}\text { Min } \\
\text { 'de' }\end{array}$ & $\begin{array}{l}\text { Li } \\
\text { 'a'/ } \\
\text { 'para }\end{array}$ & $\begin{array}{l}\text { Alaa } \\
\text { 'sobre' }\end{array}$ & $\begin{array}{l}\text { Bi } \\
\text { 'por'/ } \\
\text { 'con' }\end{array}$ & $\begin{array}{l}\text { An } \\
\text { 'alejándos } \\
\text { e' }\end{array}$ & $\begin{array}{l}\text { Otr } \\
\text { a }\end{array}$ \\
\hline $\begin{array}{l}\text { A N } \\
\text { tot } / 100 \%\end{array}$ & $26.29 \%$ & $23.47 \%$ & $23.94 \%$ & $6.10 \%$ & $\begin{array}{l}18 \\
8.45 \%\end{array}$ & $\begin{array}{l}17 \\
7.98 \%\end{array}$ & $\begin{array}{l}4 \\
1.87 \%\end{array}$ & \begin{tabular}{l}
$4.87 \%$ \\
\hline
\end{tabular} & - \\
\hline
\end{tabular}

Tabla 3. Correspondencia de la preposición $a$ en el registro culto

El porcentaje de la correspondencia en el registro culto se diferencia del registro popular como demuestran las tablas 5 y 6 . En la primera, la omisión ocupa el $26 \%$ frente a la correspondencia con la ila 'a' y con la preposición $f i$ 'en' con un porcentaje del $23 \%$ para cada una, mientras que con las otras preposiciones se disminuye bastante el porcentaje.

\begin{tabular}{|l|l|l|l|l|l|l|l|l|l|}
\hline $\begin{array}{l}\text { Registro } \\
\text { culto }\end{array}$ & $\varnothing$ & Ila & $\begin{array}{l}\text { Fi } \\
\text { 'en' }\end{array}$ & $\begin{array}{l}\text { Min } \\
\text { 'de' }\end{array}$ & $\begin{array}{l}\text { Li } \\
\text { 'a'l } \\
\text { 'para } \\
\text { 'para }\end{array}$ & $\begin{array}{l}\text { Alaa } \\
\text { 'sobre' }\end{array}$ & $\begin{array}{l}\text { Bi } \\
\text { 'por' } \\
\text { / } \\
\text { 'con' }\end{array}$ & $\begin{array}{l}\text { An } \\
\text { 'alejándos } \\
\text { e' }\end{array}$ & $\begin{array}{l}\text { Otr } \\
\text { a }\end{array}$ \\
\hline $\begin{array}{l}\text { A N } \\
\text { tot/100\% }\end{array}$ & $\begin{array}{l}295 \\
69.73 \%\end{array}$ & - & $\begin{array}{l}21 \\
4.96 \%\end{array}$ & $\begin{array}{l}2 \\
0.4 .72 \%\end{array}$ & $\begin{array}{l}61 \\
14.42 \%\end{array}$ & $\begin{array}{l}21 \\
4.96 \%\end{array}$ & $\begin{array}{l}16 \\
3.78 \%\end{array}$ & - & 7 \\
\hline
\end{tabular}

Tabla 4. Correspondencia de la preposición $a$ en el registro popular

El registro popular, presenta un porcentaje altísimo de omisión que llega al $69 \%$, mientras que la correspondencia con $L i$ 'a / para' es del $14 \%$ frente a los demás casos que no superan el $4 \%$.

Según estas estadísticas, es más probable que los alumnos egipcios cometan un porcentaje alto de errores de tipo omisión, y luego, confusión con las preposiciones en y de.

1.3.1. Comentario sobre la correspondencia de la preposición ila 'a' en la versión española de Taxi 


\section{Coincidencia absoluta con la preposición $a$ en español}

La preposición ila mantiene la correspondencia con la preposición $a$ en español en lo que se refiere a su significado como final del destino:

a) Este final del destino en árabe puede ser un lugar:

5. Ga'ah hadizan ila al kahera.

Ir [PAS $3^{\text {a }}$ PS] recientemente a el Cairo 'Había llegado recientemente a El Cairo.'

b) puede ser algo abstracto, una cosa, un objeto:

6. úhdi haza al ketab ila al hayah. (p.6)

Dedicar [PRES $1^{\text {a }}$ PS] este el libro a la vida. 'Dedico este libro a la vida.' (p.5)

7. El Nazar

Mirar [INFINTIVO]

ila al niil. (p. 97)

'Contemplar el Nilo.' (p. 63)

En español sería erróneo el empleo de "mirar a" si seguimos la traducción literal, ya que la preposición $a$ debe transmitir el límite exacto de la dirección: algo que no se puede hacer en esta situación, por lo cual resultaría adecuado el uso de la preposición hacia.

c) puede ser una persona:

8. Nazar al sa'ek ila waladayah. (p.81)

Mirar[PAS $3^{\text {a }}$ PS] el conductor a hijos-mis

El conductor miró a mis dos hijos.' (p. 51)

Equivalencia con la preposición en:

La correspondencia de la preposición ila con la preposición en se repite frecuentemente con la estructura del verbo "convertirse":

9. Kif tahawal Cómo convertir[PAS $3^{\text {a }}$ PS] este el sueño a pesadilla '¿Cómo se ha convertido este sueño en una pesadilla?' (p.13)

\footnotetext{
${ }^{1}$ Utilizamos la edición de Khaled El Khamessi, Taxi, Dar El Shorouq, 2006, p. 65 y la traducción de Alberto Canto García, Khaled Musa Sánchez, trad. Taxi, 2009, p. 42 para citar los ejemplos que utilizamos en el análisis contrastivo.
} 
El hablante árabe utiliza la preposición ila, ya que concibe el cambio como un traslado de una fase a otra, por lo tanto ila le lleva al destino final, mientras que el hablante español percibe la realidad como una ubicación interna en una situación o un estado. La preposición en según varios lingüistas de la lengua española entre ellos Schmidely (1979:169) "representa un movimiento hacia un término de doble limite y el paso de unos de los límites; en fin de movimiento, En expresa la localización dentro del límite doble." En la figura 5 se explicita la postura del hablante ante la realidad que recibe:

Hablante árabe: ¿Cómo se ha convertido el sueño a una pesadilla Sueño $\longrightarrow$ pesadilla

Hablante español: ¿Cómo se ha convertido el sueño en una pesadilla?

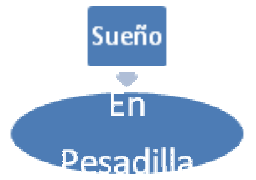

Figura 1. Representación mental del hablante

\section{Equivalencia con la preposición hacia:}

10. Inharafna $\quad$ ila share'a Ramses. (p. 36)
Desviar[PAS 2 ${ }^{\text {a }}$ PS] a calle Ramses.
'Nos desviamos hacia la calle Ramses.' (p.24)

Según López (1970: 168), aquí se utiliza la preposición hacia en vez de la preposición $a$ ya que lo que expresa hacia es "un movimiento en la dirección de una zona que engloba el lugar citado." Para Moliner (1988:156), hacia indica la iniciación del movimiento mientras que $a$ sería la llegada al objeto. Aplicando esta teoría sería más oportuno el uso de hacia en la traducción al español como correspondencia a la preposición ila que pone el acento en el final del destino.

Equivalencia con la preposición hasta:

La preposición ila en árabe a veces equivale en español a la preposición hasta en la estructura desde/ hasta:

11. Bed'a men al-omi ila al hasel ala al machester. (p. 11)

Empezando de los analfabetos hasta el obtenido sobre el máster.

'Desde los analfabetos hasta el que ha obtenido un máster.' (p.8)

Según Trujillo (1971:266-268), la preposición hasta significa "el término absoluto del movimiento", pero también incluye el rasgo de extensión del 
movimiento. Este último rasgo caracteriza la preposición desde que denota "alejamiento de un límite", por lo cual no podemos emplear ni la preposición de cuyo significado principal es "origen" ni la preposición $a$ cuya denotación es "punto final" por carecer de esta noción de extensión. No obstante, en la lengua árabe se usa ila porque indica "límite".

Para concluir, el significado de ila y el de $a$ coinciden: "en dirección a" como punto final. A pesar de ello existen algunas diferencias de matices como han demostrado los ejemplos anteriores y que se sintetizan en lo siguiente:

a) en árabe el significado de ila 'a' coincide principalmente con la preposición $a$ en español, pero a veces a este último se le añade el factor de extensión del movimiento o la variedad de direcciones, lo que hace que se conmute con las preposiciones hasta y hacia.

b) la realidad concebida en árabe como punto final, en español se interpreta como interioridad como por ejemplo: convertirse, transforma, etc.

1.3.2. Comentario sobre la correspondencia de la preposición a en la versión árabe de Taxi exponiendo los casos relevantes

\section{La correspondencia $\emptyset$}

Entre los casos más llamativos señalamos los siguientes:

a) La equivalencia $\varnothing$ en el árabe tanto en el registro culto como el coloquial en el caso del complemento directo para cosa y persona. No obstante, en español el CD suele ir precedido por la preposición $a$ aunque el grado de aparición varía si este señala a personas/ animales o a objetos:

\section{Wagadtu hafid-i. (p. 16)}

Encontrar [PAS $1^{\text {a }}$ PS] nieto-mi

'Encontré a mi nieto' (p.10)

b) La equivalencia Ø en árabe en el caso de "verbo + a + verbo" como por ejemplo: empezar a, comenzar a, principiar, etc. Segú Morera (1988:204), resulta lógico el uso de la preposición $a$ ya que su significación puntual se concuerda perfectamente con el significado de estos verbos como "inicio de un proceso":

13. Ajaz yarmi al bortukalat fy al gaw (p.7)

Empezar [PAS $3^{\text {a }}$ PS] lanzar[INFINITIVO] las naranjas en el aire 'Empezó a lanzar las naranjas al aire.' (p.6) 
Lo que se entiende en español como inicio de un proceso en árabe se interpreta como interioridad dentro de los límites de un proceso, por lo tanto se utilizaría la preposición $f i$ 'en'.

14. Yusa'ed-hu fi tajlisa-ha. (p.15)

Ayudar-le[PAS $3^{\mathrm{a}}$ PS] en solucionar-la.

'Podía ayudarle a solucionarla.' (p.10)

c) Equivalencia Ø en árabe popular con los verbos "subir, bajar, etc" mientras que en español prevalece el sentido preposicional del punto final de la preposición $a$ :

d)

15. Nazaltu al mahata. (p.182)

Bajar[PAS $1^{\text {a }}$ PS] la estación.

'Cuando bajé a la estación.' (p.114)

Correspondencia con la preposición $f i$ 'en'

Respecto a la noción temporal, la preposición $a$ en español se sustituye por $f i$ en árabe:

16. Fy awajer el shaher. (p.100)

En finales el mes.

'A finales del mes.' (p.74)

En la lengua árabe según Hussein Abid (2008: 236) la preposición $f i$ crea un espacio limitado en el cual ocurre el proceso verbal mientras que en español es imprescindible la aparición de la preposición $a$ que expresa la puntualidad temporal en estos ejemplos. Se repite lo mismo al referirse a la hora del reloj:

17. Fi el sa'ah 'asharah bilil. (p. 135)

En la hora diez noche.

'A las 10 de la noche.' (p. 79)

Correspondencia con la preposición min 'de'

a) En español lo que indicaría punto final en árabe se interpretaría como origen. Así es el caso de "miedo a/ a cercarse a":

18. Alhokuma zara'at fi-na alkawuf min algoo'a (p.24)

El gobierno sembrar[PAS $3^{\text {a }}$ PS] en-nosotros el miedo de el hambre.

El Gobierno ha sembrado en nosotros el miedo al hambre (p.16)

19. Yaktarebu min al jad. (p.88)

Acercar[PRES $3^{\text {a }}$ PS] de la raya. 
'Se acercaron a la raya.' (p. 56).

b) En situaciones que expresan igualdad o relación de comparación es habitual el uso de la preposición $a$ en español, ya que según Morera (1988:193) "la significación 'puntual' de la preposición viene pintiparado a la significación léxica de la comparación de los verbos regentes", mientras que en árabe, como aclara Lentzner (1977: 142), se utiliza la preposición $\min$ 'de' en la forma comparativa con el fin de conceptualizar el origen y aclarar el punto contrario:

20. Al mawt afdal bikazer min al kebar (p.14)

La muerte mejor mucho de la vejez.

'La muerte es preferible a la vejez.' (p.9)

\section{Correspondencia con la preposición $l i$ 'a/ para'}

Según Hussein Abid (2008:336) la preposición en árabe $l i$ significa "punto de llegada". Se distingue de la preposición ila de la siguiente manera: esta mira al punto como el fin del destino, mientras que $l i$ como asignado en particular, por lo tanto en español su equivalencia se va turnando entre $a$ y para:

a) La preposición $a$ coincide con $l i$ ante el complemento indirecto, considerándolo como la persona a quien se le dirige la acción:

21. Ashraah li wilady almawkef. (p.60)

Explicar[PRES 1 ${ }^{\text {a }}$ PS] a hijos mis la situación.

'Les estoy explicando a mis hijos la situación.' (p.38)

b) En las situaciones donde la preposición $a$ expresa el movimiento determinado con un fin incluido, en árabe muchas veces se utilizaría $l i$, concibiendo el acto como una finalidad:

22. Takadamo li jawod al intijabat. (p.139)

Presentar [PAS $3^{\text {a }}$ PS] para participación las elecciones.

'Se han presentado a las elecciones.' (p.88) 
Correspondencia con la preposición alaa 'sobre'

Según Lentzner (1977: 64) la preposición alaa en árabe significa "superioridad". En la novela hemos registrado algunos ejemplos en los cuales la realidad en la lengua árabe se concibe desde el criterio de estar por encima de aquello del que se habla.

23. Sakat ala al ard. (p. 145)

Caer [PAS $3^{\text {a }}$ PS] sobre el suelo.

'Se cayó al suelo.' (p.117)

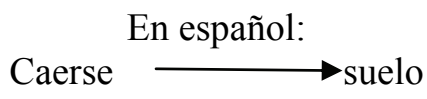

La realidad en árabe:

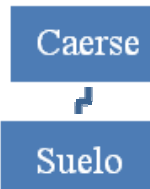

La figura 2. La realidad mental en español y en árabe

Recopilando los resultados más significativos de este apartado, subrayamos que:

- se omite la equivalencia de preposición $a$ en árabe en el caso de complemento directo, la conexión entre dos verbos que expresan el comienzo de un proceso, y en el caso de los verbos "subir, baja, etc.".

- en varias ocasiones se cambia la realidad introducida por la preposición $a$ al árabe: el movimiento con un fin determinado se entiende en árabe como:

a) una realidad de inclusión $f i$ 'en': noción del tiempo.

b) una partida del punto de origen $\min$ 'de': acercarse, tener miedo, relaciones de comparaciones, etc.

c) un acto de finalidad $l i$ 'a/ para': complemento indirecto, situaciones donde se interpreta el fin de la dirección como una finalidad en sí: invitar a, dedicarse a, presentarse a, etc.

\section{ANÁLISIS DE ERRORES: PRESENTACIÓN DE LA INVESTIGACIÓN}

Nuestra investigación empírica tiene como objetivo analizar los errores de la preposición $a$ en la expresión escrita de alumnos egipcios con el fin de mejorar la enseñanza de las preposiciones dentro de la programación de los cursos de español como L2. Nos acercamos a la interlengua de unos alumnos de dos niveles distintos, intermedio y superior, para averiguar la persistencia de los errores del uso de la preposición $a$ en las últimas etapas del aprendizaje. La herramienta empleada en 
este apartado incluye: 1. un cuestionario sobre el historial de las lenguas extranjeras del corpus de datos; 2 . una tarea de redacción de 30 minutos.

\subsection{Material de la muestra (tema de composición)}

Se ha asignado una tarea de redacción a los dos grupos antes mencionados, basada en un tema general y abierto sobre el cine. La extensión de palabras fue libre en un periodo de tiempo dividido de la siguiente manera: en 5 minutos cada alumno tenía que determinar la película sobre la cual escribiría un comentario y en los 25 minutos restantes debía redactar la composición. Las instrucciones se daban en español y nuestro papel se limitaba a orientar en cuanto a las pautas. La redacción se escribía completamente en español excepto el título de la película que se podía apuntar en la lengua de origen de la misma.

\subsection{Toma de datos}

Se han recopilado datos sincrónicos que analizan un estadio concreto de la interlengua de unos alumnos y eso se debe a la limitación del tiempo de nuestra investigación que nos impide seguir el desarrollo del aprendizaje en varios intervalos periódicos. El tamaño total de la muestra es de 60 redacciones recogidas a finales del segundo semestre del año académico 2013. 


\subsection{Descripción del corpus}

- Los informantes ${ }^{2}$ : en total dos grupos de estudiantes de 30 alumnos cada uno. Todos ellos comparten la misma lengua materna: el árabe. Son perfiles de alumnos universitarios que están cursando el segundo y el cuarto año de Filología Hispánica. La edad de estos estudiantes está comprendida entre 17 y 19 años. Se trata de una muestra heterogénea en lo que se refiere a edad y sexo; no obstante, se presenta una mayoría femenina: 42 mujeres.

- Centro seleccionado: Departamento de Filología Hispánica, en la Universidad de Helwan, Egipto. La selección del centro se realizó atendiendo a dos circunstancias:

- El número de los candidatos en la sede era lo suficientemente amplio para extraer de ella los individuos necesarios para conformar la muestra de nuestra investigación.

- El centro está situado en un contexto académico extranjero en el cual el español es un medio y fin al mismo tiempo.

${ }^{2}$ Según unos cuestionarios manejados en la investigación con el fin de informarnos del historial de las lenguas que dominan los dos grupos de alumnos investigados, nos percatamos de que el $90 \%$ de los informantes del primer grupo (segundo año de carrera), de nivel A2- B1, comparte el inglés como primera lengua extranjera frente a $10 \%$ cuyo primer contacto con lenguas europeas era a través del español, impartido durante la etapa universitaria. Respecto al inglés el grado de dominio es variado, pero el nivel regular ocupa el porcentaje más amplio $40 \%$. En cuanto a las demás lenguas, el francés posee el $25 \%$ frente al alemán y el italiano con un porcentaje que no supera el 10\%. El nivel general de dominio de estas lenguas es regular. El periodo de aprendizaje del español ha sido 3 años. Ninguno de los alumnos ha estado en un país hispanohablante, pero esto no significa que no haya contacto con nativos. El $70 \%$ practican el español con nativos a través de las redes sociales o un sistema de intercambio lingüístico que fomenta el Instituto Cervantes en El Cairo. Igual que la mayoría del grupo anterior los informantes del segundo grupo (cuarto año de carrera), de nivel B2-C1, han estudiado el inglés como primera lengua extranjera. Su dominio vacila entre regular con un porcentaje del $24 \%$ frente al $52 \%$ con un nivel bueno. En cuanto a las otras lenguas, el francés ocupa el 54\% mientras que las demás entre $15 \%$ y $5 \%$ con un nivel lingüístico regular. El periodo de enseñanza de la lengua española en la mayoría no supera los 4 años de carrera. Por último, el 44\% de estos alumnos pone en práctica lo que ha aprendido en español a través del chat en Facebook, Twitter o a través de contacto directo con españoles o latinos residentes en El Cairo frente al 48\% que no tiene ningún tipo de comunicación con hispanohablantes. Este porcentaje debería ser un poco alarmante, ya que afectará al desarrollo de la interlengua y su acercamiento a la lengua objeto (LO). 


\subsection{Presentación de los resultados del análisis de errores}

Antes de presentar los resultados del análisis de errores nos parece oportuno aclarar que el departamento de Filología Hispánica de la universidad de Helwan se considera bastante joven. El cuarto curso de carrera es la primera promoción que se gradúa desde la inauguración del departamento en 2010. Una de las profesoras titulares del curso nos ha comentado que esta última promoción posee un nivel inferior a lo habitual ya sea por falta de interés por parte de los alumnos, que no ven mucho futuro laboral aprendiendo español, y también por falta de recursos y plantilla en el departamento en aquel entonces. En cambio, la promoción del segundo año disfruta de mejores recursos y están más motivados. La mayoría aspira a obtener una beca para viajar a un país hispanohablante y a trabajar en empresas españolas dentro o fuera del país.

Comenzamos con el análisis de los datos del primer grupo ( $2^{\circ}$ curso). En total hemos analizado 30 redacciones con una extensión de 9-15 líneas. Las siguientes tablas exponen la estadística del porcentaje del uso correcto y anómalo de la preposición $a$ en las muestras:

El resultado del primer grupo ( $2^{\circ}$ curso $)$

\begin{tabular}{|c|c|c|c|c|}
\hline $\begin{array}{l}\text { Tipo } \\
\text { de errores }\end{array}$ & personal & de $a$ Ausencia & $\begin{array}{l}\text { Falsa } \\
\text { selección }\end{array}$ & $\begin{array}{l}\text { Errores } \\
\text { de adición }\end{array}$ \\
\hline $\begin{array}{l}\text { Uso } \\
\text { anómalo } \\
\text { Total: } \\
\text { T9/59\% }\end{array}$ & $29 / 49 \%$ & $8 / 13 \%$ & $12 / 22 \%$ & $10 / 16.9 \%$ \\
\hline & personal & \multicolumn{3}{|c|}{ Colocación correcta } \\
\hline $\begin{array}{l}\text { Uso } \\
\text { correcto } \\
41 / 41 \%\end{array}$ & $15 / 34 \%$ & \multicolumn{3}{|l|}{$26 / 62 \%$} \\
\hline $\begin{array}{l}\text { Total: } \\
100 / 100 \%\end{array}$ & $44 / 100 \%$ & \multicolumn{3}{|l|}{$56 / 100 \%$} \\
\hline
\end{tabular}

Tabla 5. El resultado de las muestras del grupo I

En la tabla de arriba, el uso anómalo se clasifica en varias subcategorías. Entre ellas observamos que el porcentaje más alto de errores pertenece al uso de apersonal frente a los demás tipos que siguen el sucesivo orden estadísticamente: falsa selección $22 \%$, errores de adición $16.9 \%$, y ausencia de a $13 \%$. El uso correcto se ha divido en a- personal con $34 \%$ y la colocación correcta con $62 \%$.

Basándonos en los criterios adaptados de Graciela Vázquez (1998:25), los criterios lingüísticos serán el eje que categorizan los errores preposicionales 
detectados en las muestras. Dichos criterios se dividen en: errores de adición, errores de omisión, errores de yuxtaposición, errores de falsa colocación y errores de falsa selección, cuya definición según la autora (1998:32) viene explicada a continuación junto a los ejemplos detectados en cada caso:

Falsa selección: consiste en seleccionar morfemas o palabras incorrectas en un contexto determinado.

- En vez de $a$ seleccionan en:

24. Fui en la escuela

25. Le llevan en Istampul.

- Utilizar $a$ en vez de otras preposiciones:

26. Puede huir a la muerte

27. El autor concentra a los sueños.

Adición: consiste en añadir morfemas o palabras que son redundantes.

28. No expresa al idea

29. Tenemos que planear a nuestro sueño

30. Tiene un capital para empezar al negocio.

31. Empezaron a su viaje.

Omisión: consiste en eliminar morfemas o palabras que no son redundantes.

- omisión entre dos verbos:

32. Comenzaron vender.

33. volvió escapar.

- $\quad$ omisión de la preposición $a$ ante el CD:

34. Amé un chico

35. Sabía un chico

36. Encontré un hombre

37. Quise un chico

38. Ví un hombre

En esta fase de aprendizaje, el alumno se apoya más en su lengua materna, por lo cual, no nos sorprende la abundancia de errores por falta de dominio de las estructuras de la lengua que se está aprendiendo o por interferencia negativa con la L1. Como consecuencia del primer caso, aparecen los errores de confusión entre la en y $a$ en los ejemplos (24-27); y por otro lado, los errores de adición en los ejemplos (28-31) que se han producido por generalizar algunas reglas de L2 como la de "empezar + a": empezaron a su viaje. Este tipo de errores desaparecerán en etapas posteriores del aprendizaje.

En cuanto a la interferencia negativa, el porcentaje más alto de errores se encuentra en el caso de omisión con el CD y otro más reducido en algunos casos de la estructura de la preposición $a$ entre dos verbos. Los resultados del análisis contrastivo, cotejando la correspondencia de la preposición $a$ en español con su homóloga ila 'a' en árabe, nos ha mostrado que en la lengua árabe no se le añade ninguna preposición al complemento directo ni se utiliza con mucha frecuencia la 
preposición ila 'a' para conectar dos verbos. En este último caso se omite el conector ya que el vínculo se sobreentiende, pero aún así en las situaciones en las que sí hace falta un nexo preposicional entre verbos, la alternativa suele ser la preposición $f i$ 'en'. Esto justifica la aparición frecuente de dichos errores y nos incita a estar atentos ante la posibilidad de desarrollar un error fosilizable que nos lo va a confirmar el resultado del análisis del segundo grupo de nivel B2- C1.

El resultado del análisis de los datos del segundo grupo ( $4^{\circ}$ curso)

El tamaño de las muestras incluye 30 redacciones de extensión de 9-15 líneas.

\begin{tabular}{|l|c|c|c|c|}
\hline $\begin{array}{c}\text { Tipo de } \\
\text { errores }\end{array}$ & a-personal & $\begin{array}{c}\text { Ausencia } \\
\text { de } a\end{array}$ & $\begin{array}{c}\text { Falsa } \\
\text { selección }\end{array}$ & $\begin{array}{c}\text { Errores de } \\
\text { adición }\end{array}$ \\
\hline $\begin{array}{c}\text { Uso anómalo } \\
\text { Total: } \\
37 / 37 \%\end{array}$ & $20 / 54 \%$ & $5 / 13 \%$ & $7 / 18 \%$ & $5 / 13 \%$ \\
\hline \hline & a-personal & \multicolumn{2}{|c|}{ Colocación correcta } \\
\hline $\begin{array}{c}\text { Uso correcto } \\
\text { Total: } \\
63 / 63 \%\end{array}$ & $26 / 41.2 \%$ & $37 / 58.7 \%$ & \\
\hline $\begin{array}{l}\text { Total: } \\
100 / 100 \%\end{array}$ & $46 / 100 \%$ & $54 / 100 \%$ & \\
\hline
\end{tabular}

Tabla 6. El resultado de las muestras del grupo II

Esta tabla sigue la misma clasificación que la tabla 7. Aquí el uso anómalo de a-personal ocupa el porcentaje mayoritario con el $54 \%$ en comparación con los demás: falsa selección $18 \%$, ausencia de a 13\%, y errores de adición $13 \%$. En lo que se refiere al uso correcto, la colocación correcta apunta el porcentaje 58.7\% frente al a-personal con $41.2 \%$.

Aplicando los mismos criterios que la sección anterior, los errores preposicionales más frecuentes se clasifican en:

\section{Falsa selección:}

- $\quad$ En vez de $a$ seleccionan en:

39. En final logró su objetivo.

40. El protagonista descubrió en lo lejos.

41. Comenzó en ver la película.

- Utilizar $a$ en vez de otras preposiciones

Omisión:

42. Se convirtió a una persona responsable.

- Omisión de la preposición $a$ ante el CD (área fosilizable):

43. Ama chico.

44. Trata su hermana. 
45. Encuentra una persona.

En este grupo de nivel avanzado el porcentaje del error en general se ha disminuido en un $22 \%$. No obstante, la omisión de la preposición a con el CD sigue agrupando el conjunto mayor de errores. En total, ha persistido el 37\% hasta la última etapa de la carrera universitaria, lo cual da pie al fenómeno del error fosilizable. En una misma redacción se alternan el uso correcto de la preposición con el CD y el uso anómalo de omisión, lo que refleja la práctica forzada que hace el alumno para asimilar esta estructura de LO que no existe en el sistema de su lengua materna.

Para concluir, el uso incorrecto de la preposición con el CD es un problema no solamente de alumnos de origen árabe sino de diferentes nacionalidades. Dentro de la gramática española hallamos muchos estudios relevantes sobre la preposición $a$ y el CD. Nuestro estudio no puede adentrarse en ello, pero exponemos una explicación de E. A. Llorch (1995: 229) al respecto: la función de objeto directo carece en principio de índice funcional, pero si la unidad que la cumple hace referencia a entes capaces de ser actores de la noción designada por el verbo, la necesidad de distinguirlo del sujeto impone en el objeto directo la preposición $a$.

El resultado de nuestra investigación debería inducirnos a reflexionar como docentes sobre cómo trabajar esta parcela gramatical de tal manera que reduzca el porcentaje de errores. Es preferible remediar los errores en etapas iniciales del aprendizaje ya que con el tiempo la motivación del alumno por aprender las formas correctas de LO se reduce según nos confirma Corder (1984:64): "una vez que la gramática de la IL del aprendiz está lo suficientemente desarrollada para permitirle una comunicación adecuada para sus propósitos disminuye la motivación para mejorar".

\section{CONCLUSIÓN}

En nuestra investigación se ha intentado mostrar las peculiaridades que manifiesta la interlengua de unos aprendices árabes en una fase determinada de su aprendizaje de ELE. Entre los más notables destacan aquellos que afectan a la construcción de las oraciones de la preposición $a$. Se hace énfasis sobre el fenómeno de la transferencia de la lengua materna que, a su vez, incide en las desviaciones de la interlengua de los aprendices

Según los resultados de la investigación, es muy frecuente la omisión de la preposición $a \mathrm{y}$ en menor medida la confusión entre la $a \mathrm{y}$ otras preposiciones. El porcentaje del uso anómalo de la preposición $a$ en nivel intermedio ha sido 59\% frente a $37 \%$ en niveles superiores. Los errores, en general, disminuyen con el avance del aprendizaje, pero aún así algunos de ellos persisten incluso en las últimas etapas de enseñanza/aprendizaje como por ejemplo la omisión de la preposición $a$ ante el CD.

Para terminar, esperamos que aumente la bibliografía y estudios sobre la interlengua de aprendices árabes como es el caso de otros grupos lingüísticos 
(anglófonos, francófonos, italianos, etc.) para poder tratar otras cuestiones que nuestro trabajo no ha cubierto por varias razones, entre ellas, la falta de tiempo.

\section{BIBLIOGRAFÍA}

ALARCOS LLORACH, Emilio (1995): Gramática de la Lengua Española, Madrid, Espasa Calpe.

CANTÓ GARCÍA, Alberto y Khaled Musa SÁNCHEZ, (2009): trad. Taxi, Barcelona, Almuzara.

CONSEJO DE EUROPA (2002): Marco Común Europeo de Referencia para las Lenguas: aprendizaje, enseñanza, evaluación, Madrid, Ministerio de Educación, Cultura y Deporte y Grupo Anaya.

CORDER, S. Pit (1981): "The significance of learners'errors", en Error Analysis and interlanguage, Oxford University, 5-11.

EL KHAMESSI, Khaled (2006): Taxi, El Cairo, Dar El Shorouq..

FERNÁNDEZ LÓPEZ, Sonsoles (1995): "Errores e interlengua en el aprendizaje del español como lengua española”, Didáctica, n ${ }^{\circ}$ 7, 204.

HUSSEIN ABID, Abeer (2008): Tesis Doctoral: El esquema básico de la referencia. Un modelo del significado esquemático aplicado a las preposiciones españolas y árabes, Universidad de Alicante.

JAMES, Carl (1998): Errors in Language Learning and Use, Sideny University.

KRASHEN, Stephen. (1982): Principles and Practices in Second Language Acquisitions, Oxford, Pergamón.

LARSEN- FREEMAN, Diana y LONG, Michael (1984): Introducción al estudio de la adquisición de segundas lenguas, Madrid,Gredos

LENTZNER, Karin (1977): Semantic and Syntactic Aspects of Arabic Prepositions, Georgetown Univeristy.

LÓPEZ, M. L. (1970): Problemas y Métodos en El análisis de Preposiciones, Madrid, Gredos.

MILLAR, María Angélica, Rosa SALGADO, Marcela ZEDÁN (2005): Gramática de la Lengua árabe para hispanohablante, Universidad de Chile.

MORERA, Marcial (1988): Estructura semántica del sistema preposicional del español moderno y sus campos de usos, Servicio de Publicaciones del Cabildo Insular de Fuerteventura.

RICHARDS. JACK C. y Theodore S. RODGERS (2003): Enfoques y métodos en la enseñanza de idiomas, Cambridge Uuniversity press.

Real Academia Española http://lema.rae.es/drae/?val=preposicion [consulta 10 mayo: 2013]

Real Academia Española (2010): Nueva Gramática de la Lengua Española, Espasa, Madrid. 
SANTOS GARGALLO, Isabel (2004): "El análisis de errores en la interlingua de un hablante no nativo", en Vademécum para la formación de profesores, J. Sánchez Lobato e I. Santos Gargallo (Coord.), Madrid, SGEL, 393-394.

SELINKER, Larry (1974): "Interlanguage", en Error Analysis: Perspectives on Second Language Acquisition, Jack C. Richards y Gloria P Sampson (Coord), London, Longman, 31-55.

SCHMIDELY, Jack (1979): "Preposiciones Españolas: De "En "A "contra", en Lingüistica Española Actual, n. 12, 169.

TRUJILLO, Ramón (1971): "Notas para un estudio de las preposiciones españolas" Thesaurus. N ${ }^{\circ} 26,237-268$.

VÁZQUEZ, Graciela (1998): Error sin falta, Madrid, Edelsa.

WEINREICH, Uriel (1968): Languages in Contact, Paris, Mouton 